\title{
FIXED POINTS AND STABILITY IN NEUTRAL DIFFERENTIAL EQUATIONS WITH VARIABLE DELAYS
}

\author{
CHUHUA JIN AND JIAOWAN LUO
}

(Communicated by Carmen C. Chicone)

\begin{abstract}
In this paper we consider a linear scalar neutral delay differential equation with variable delays and give some new conditions to ensure that the zero solution is asymptotically stable by means of fixed point theory. These conditions do not require the boundedness of delays, nor do they ask for a fixed sign on the coefficient functions. An asymptotic stability theorem with a necessary and sufficient condition is proved. The results of Burton, Raffoul, and Zhang are improved and generalized.
\end{abstract}

\section{INTRODUCTION}

Lyapunov's direct method has been very effective in establishing stability results for a wide variety of differential equations. Yet, there is a large set of problems for which it has been ineffective. Recently, Burton and others applied fixed point theory to study stability [2-9]. It has been shown that many of those problems encountered in the study of stability by means of Lyapunov's direct method can be solved by using fixed point theory. While Lyapunov's direct method usually requires pointwise conditions, the stability result by fixed point theory asks conditions of an averaging nature.

In the present paper we also adopt fixed point theory to study the asymptotic stability of neutral delay differential equations. A new technique is used, which makes stability conditions more feasible and the results in $[3,8,9]$ are improved and generalized.

The rest of this paper is organized as follows. In Section 2, we state some known results and our main theorem; the proof of our result is also given in this section. In Section 3, two examples show that our stability result, not only for delay differential equations but also for neutral delay differential equations, is indeed better than those in $[3,8,9]$.

Received by the editors October 10, 2006.

2000 Mathematics Subject Classification. Primary 34K20, 34K40.

Key words and phrases. Fixed points, stability, neutral delay differential equations, variable delays.

The second author was supported in part by NNSF of China Grant \#10671043. 


\section{MAIN RESUlT}

Consider the following neutral delay differential equations with variable delays of the form

$$
x^{\prime}(t)=-a(t) x(t)-b(t) x(t-\tau(t))+c(t) x^{\prime}(t-\tau(t)),
$$

where $a, b, c \in C\left(R^{+}, R\right)$ and $\tau \in C\left(R^{+}, R^{+}\right)$with $t-\tau(t) \rightarrow \infty$ as $t \rightarrow \infty$.

Equation (2.1) and its special cases have been investigated by many authors. For example, Burton in [3] and Zhang in [9] have studied the equation

$$
x^{\prime}(t)=-b(t) x(t-\tau(t))
$$

and obtained the following.

Theorem A (Burton [3]). Suppose that $\tau(t)=r$ and there exists a constant $\alpha<1$ such that

$$
\int_{t-r}^{t}|b(s+r)| d s+\int_{0}^{t}|b(s+r)| e^{-\int_{s}^{t} b(u+r) d u} \int_{s-r}^{s}|b(u+r)| d u d s \leq \alpha
$$

for all $t \geq 0$ and $\int_{0}^{\infty} b(s) d s=\infty$. Then for every continuous initial function $\psi:[-r, 0] \rightarrow R$, the solution $x(t)=x(t, 0, \psi)$ of $(2.2)$ is bounded and tends to zero as $t \rightarrow \infty$.

Theorem B (Zhang [9]). Suppose that $\tau$ is differentiable, the inverse function $g(t)$ of $t-\tau(t)$ exists, and there exists a constant $\alpha \in(0,1)$ such that for $t \geq 0$

$$
\begin{gathered}
\liminf _{t \rightarrow \infty} \int_{0}^{t} b(g(s)) d s>-\infty, \\
\int_{t-\tau(t)}^{t}|b(g(s))| d s+\int_{0}^{t} e^{-\int_{s}^{t} b(g(u)) d u}|b(g(s))| \int_{s-\tau(s)}^{s}|b(g(v))| d v d s \\
+\int_{0}^{t} e^{-\int_{s}^{t} b(g(u)) d u}|b(s)|\left|\tau^{\prime}(s)\right| d s \leq \alpha .
\end{gathered}
$$

Then the zero solution of (2.2) is asymptotically stable if and only if

$$
\int_{0}^{t} b(g(s)) d s \rightarrow \infty \quad \text { as } \quad t \rightarrow \infty
$$

Obviously, Theorem B improves Theorem A. On the other hand, Raffoul in [8] has investigated equation (2.1) and obtained

Theorem C (Raffoul [8]). Let $\tau(t)$ be twice differentiable and $\tau^{\prime}(t) \neq 1$ for all $t \in R$. Suppose that there exists a constant $\alpha \in(0,1)$ such that for $t \geq 0$

$$
\int_{0}^{t} a(s) d s \rightarrow \infty \quad \text { as } \quad t \rightarrow \infty
$$

and

$$
\left|\frac{c(t)}{1-\tau^{\prime}(t)}\right|+\int_{0}^{t} e^{-\int_{s}^{t} a(u) d u}\left|b(s)+\frac{\left[c(s) a(s)+c^{\prime}(s)\right]\left(1-\tau^{\prime}(s)\right)+c(s) \tau^{\prime \prime}(s)}{\left(1-\tau^{\prime}(s)\right)^{2}}\right| d s \leq \alpha .
$$

Then every solution $x(t)=x(t, 0, \psi)$ of $(2.1)$ with a small continuous initial function $\psi(t)$ is bounded and tends to zero as $t \rightarrow \infty$. 
Let $R=(-\infty,+\infty), R^{+}=[0,+\infty)$, and $R^{-}=(-\infty, 0]$, respectively. $C\left(S_{1}, S_{2}\right)$ denotes the set of all continuous functions $\phi: S_{1} \rightarrow S_{2}$. For each $t_{0}$, define $m\left(t_{0}\right)=$ $\inf \left\{s-\tau(s): s \geq t_{0}\right\}$, and $C\left(t_{0}\right)=C\left(\left[m\left(t_{0}\right), t_{0}\right], R\right)$ with the supremum norm $\|\cdot\|$.

For each $\left(t_{0}, \phi\right) \in R^{+} \times C\left(t_{0}\right)$, a solution of $(2.1)$ through $\left(t_{0}, \phi\right)$ is a continuous function $x:\left[m\left(t_{0}\right), t_{0}+\alpha\right) \rightarrow R^{n}$ for some positive constant $\alpha>0$ such that $x(t)$ satisfies $(2.1)$ on $\left[t_{0}, t_{0}+\alpha\right)$ and $x(s)=\phi(s)$ for $s \in\left[m\left(t_{0}\right), t_{0}\right]$. We denote such a solution by $x(t)=x\left(t, t_{0}, \phi\right)$. For each $\left(t_{0}, \phi\right) \in R^{+} \times C\left(t_{0}\right)$, there exists a unique solution $x(t)=x\left(t, t_{0}, \phi\right)$ of $(2.1)$ defined on $\left[t_{0},+\infty\right)$. For fixed $t_{0}$, we define $\|\phi\|=\max \left\{|\phi(s)|: m\left(t_{0}\right) \leq s \leq t_{0}\right\}$. Stability definitions may be found in [1], for example.

Theorem 2.1. Let $\tau(t)$ be twice differentiable and $\tau^{\prime}(t) \neq 1$ for all $t \in R$. Suppose that there exists a constant $\alpha \in(0,1)$ and a function $h \in C\left(R^{+}, R\right)$ such that for $t \geq 0$

(i)

$$
\liminf _{t \rightarrow \infty} \int_{0}^{t} h(s) d s>-\infty
$$

(ii)

$$
\begin{aligned}
& \left|\frac{c(t)}{1-\tau^{\prime}(t)}\right|+\int_{t-\tau(t)}^{t}|h(s)-a(s)| d s \\
& +\int_{0}^{t} e^{-\int_{s}^{t} h(u) d u} \mid-b(s)+[h(s-\tau(s))-a(s-\tau(s))]\left(1-\tau^{\prime}(s)\right) \\
& \quad-\frac{\left[c(s) h(s)+c^{\prime}(s)\right]\left(1-\tau^{\prime}(s)\right)+c(s) \tau^{\prime \prime}(s)}{\left(1-\tau^{\prime}(s)\right)^{2}} \mid d s \\
& +\int_{0}^{t} e^{-\int_{s}^{t} h(u) d u}|h(s)| \int_{s-\tau(s)}^{s}|h(v)-a(v)| d v d s \leq \alpha .
\end{aligned}
$$

Then the zero solution of (2.1) is asymptotically stable if and only if (iii)

$$
\int_{0}^{t} h(s) d s \rightarrow \infty \quad \text { as } \quad t \rightarrow \infty
$$

Proof. First, suppose that (iii) holds. For each $t_{0} \geq 0$, we set

$$
K=\sup _{t \geq 0}\left\{e^{-\int_{0}^{t} h(s) d s}\right\} .
$$

Let $\phi \in C\left(t_{0}\right)$ be fixed and define

$S=\left\{x \in C\left(\left[m\left(t_{0}\right), \infty\right), R\right): x(t) \rightarrow 0\right.$ as $t \rightarrow \infty, x(s)=\phi(s)$ for $\left.s \in\left[m\left(t_{0}\right), t_{0}\right]\right\}$.

Then $S$ is a complete metric space with metric $\rho(x, y)=\sup _{t \geq t_{0}}\{|x(t)-y(t)|\}$.

Multiply both sides of (2.1) by $e^{\int_{t_{0}}^{t} h(s) d s}$ and then integrate from $t_{0}$ to $t$ to obtain

$$
\begin{aligned}
x(t) & =\phi\left(t_{0}\right) e^{-\int_{t_{0}}^{t} h(s) d s}+\int_{t_{0}}^{t} e^{-\int_{s}^{t} h(u) d u}[h(s)-a(s)] x(s) d s \\
& -\int_{t_{0}}^{t} e^{-\int_{s}^{t} h(u) d u} b(s) x(s-\tau(s)) d s+\int_{t_{0}}^{t} e^{-\int_{s}^{t} h(u) d u} c(s) x^{\prime}(s-\tau(s)) d s .
\end{aligned}
$$


Performing an integration by parts, we have

$$
\begin{aligned}
x(t)= & \phi\left(t_{0}\right) e^{-\int_{t_{0}}^{t} h(s) d s}+\int_{t_{0}}^{t} e^{-\int_{s}^{t} h(u) d u} d\left(\int_{s-\tau(s)}^{s}[h(v)-a(v)] x(v) d v\right) \\
& +\int_{t_{0}}^{t} e^{-\int_{s}^{t} h(u) d u}\left\{-b(s)+[h(s-\tau(s))-a(s-\tau(s))]\left(1-\tau^{\prime}(s)\right)\right\} \\
& \times x(s-\tau(s)) d s+\int_{t_{0}}^{t} \frac{c(s)}{1-\tau^{\prime}(s)} e^{-\int_{s}^{t} h(u) d u} d x(s-\tau(s)) \\
= & \left\{\phi\left(t_{0}\right)-\frac{c\left(t_{0}\right)}{1-\tau^{\prime}\left(t_{0}\right)} \phi\left(t_{0}-\tau\left(t_{0}\right)\right)-\int_{t_{0}-\tau\left(t_{0}\right)}^{t_{0}}[h(s)-a(s)] \phi(s) d s\right\} e^{-\int_{t_{0}}^{t} h(u) d u} \\
& +\frac{c(t)}{1-\tau^{\prime}(t)} x(t-\tau(t))+\int_{t-\tau(t)}^{t}[h(s)-a(s)] x(s) d s \\
+ & \int_{t_{0}}^{t} e^{-\int_{s}^{t} h(u) d u}\left\{-b(s)+[h(s-\tau(s))-a(s-\tau(s))]\left(1-\tau^{\prime}(s)\right)\right. \\
& \left.-\frac{\left[c(s) h(s)+c^{\prime}(s)\right]\left(1-\tau^{\prime}(s)\right)+c(s) \tau^{\prime \prime}(s)}{\left(1-\tau^{\prime}(s)\right)^{2}}\right\} x(s-\tau(s)) d s \\
& -\int_{t_{0}}^{t} e^{-\int_{s}^{t} h(u) d u} h(s) \int_{s-\tau(s)}^{s}[h(v)-a(v)] x(v) d v d s .
\end{aligned}
$$

Use (2.10) to define the operator $P: S \rightarrow S$ by $(P x)(t)=\phi(t)$ for $t \in\left[m\left(t_{0}\right), t_{0}\right]$ and

$$
\begin{aligned}
(P x)(t)=\{ & \left.\phi\left(t_{0}\right)-\frac{c\left(t_{0}\right)}{1-\tau^{\prime}\left(t_{0}\right)} \phi\left(t_{0}-\tau\left(t_{0}\right)\right)-\int_{t_{0}-\tau\left(t_{0}\right)}^{t_{0}}[h(s)-a(s)] \phi(s) d s\right\} \\
& \times e^{-\int_{t_{0}}^{t} h(u) d u}+\frac{c(t)}{1-\tau^{\prime}(t)} x(t-\tau(t))+\int_{t-\tau(t)}^{t}[h(s)-a(s)] x(s) d s \\
& +\int_{t_{0}}^{t} e^{-\int_{s}^{t} h(u) d u}\left\{-b(s)+[h(s-\tau(s))-a(s-\tau(s))]\left(1-\tau^{\prime}(s)\right)\right. \\
& \left.-\frac{\left[c(s) h(s)+c^{\prime}(s)\right]\left(1-\tau^{\prime}(s)\right)+c(s) \tau^{\prime \prime}(s)}{\left(1-\tau^{\prime}(s)\right)^{2}}\right\} x(s-\tau(s)) d s \\
& -\int_{t_{0}}^{t} e^{-\int_{s}^{t} h(u) d u} h(s) \int_{s-\tau(s)}^{s}[h(v)-a(v)] x(v) d v d s
\end{aligned}
$$

for $t \geq t_{0}$. It is clear that $(P x) \in C\left(\left[m\left(t_{0}\right), \infty\right), R\right)$. We now show that $(P x)(t) \rightarrow 0$ as $t \rightarrow \infty$. Since $x(t) \rightarrow 0$ and $t-\tau(t) \rightarrow \infty$ as $t \rightarrow \infty$, for each $\varepsilon>0$, there exists a $T_{1}>t_{0}$ such that $s \geq T_{1}$ implies that $|x(s-\tau(s))|<\varepsilon$. Thus, for $t \geq T_{1}$, the last 
term $I_{5}$ in $(2.11)$ satisfies

$$
\begin{aligned}
\left|I_{5}\right|= & \left|\int_{t_{0}}^{t} e^{-\int_{s}^{t} h(u) d u} h(s) \int_{s-\tau(s)}^{s}[h(v)-a(v)] x(v) d v d s\right| \\
\leq & \int_{t_{0}}^{T_{1}} e^{-\int_{s}^{t} h(u) d u}|h(s)| \int_{s-\tau(s)}^{s}|h(v)-a(v)||x(v)| d v d s \\
& +\int_{T_{1}}^{t} e^{-\int_{s}^{t} h(u) d u}|h(s)| \int_{s-\tau(s)}^{s}|h(v)-a(v)||x(v)| d v d s \\
\leq & \sup _{\sigma \geq m\left(t_{0}\right)}|x(\sigma)| \int_{t_{0}}^{T_{1}} e^{-\int_{s}^{t} h(u) d u}|h(s)| \int_{s-\tau(s)}^{s}|h(v)-a(v)| d v d s \\
& +\varepsilon \int_{T_{1}}^{t} e^{-\int_{s}^{t} h(u) d u}|h(s)| \int_{s-\tau(s)}^{s}|h(v)-a(v)| d v d s .
\end{aligned}
$$

By (iii), there exists $T_{2}>T_{1}$ such that $t \geq T_{2}$ implies

$$
\begin{aligned}
& \sup _{\sigma \geq m\left(t_{0}\right)}|x(\sigma)| \int_{t_{0}}^{T_{1}} e^{-\int_{s}^{t} h(u) d u}|h(s)| \int_{s-\tau(s)}^{s}|h(v)-a(v)| d v d s \\
& =\sup _{\sigma \geq m\left(t_{0}\right)}|x(\sigma)| e^{-\int_{T_{1}}^{t} h(u) d u} \int_{t_{0}}^{T_{1}} e^{-\int_{s}^{T_{1}} h(u) d u}|h(s)| \int_{s-\tau(s)}^{s}|h(v)-a(v)| d v d s<\varepsilon .
\end{aligned}
$$

Apply (ii) to obtain $\left|I_{5}\right| \leq \varepsilon+\alpha \varepsilon<2 \varepsilon$. Thus, $I_{5} \rightarrow 0$ as $t \rightarrow \infty$. Similarly, we can show that the rest of the terms in (2.11) approach zero as $t \rightarrow \infty$. This yields $(P x)(t) \rightarrow 0$ as $t \rightarrow \infty$, and hence $P x \in S$. Also, by (ii), $P$ is a contraction mapping with contraction constant $\alpha$. By the Contraction Mapping Principle, $P$ has a unique fixed point $x$ in $S$ which is a solution of $(2.1)$ with $x(s)=\phi(s)$ on $\left[m\left(t_{0}\right), t_{0}\right]$ and $x(t)=x\left(t, t_{0}, \phi\right) \rightarrow 0$ as $t \rightarrow \infty$.

To obtain asymptotic stability, we need to show that the zero solution of (2.1) is stable. Let $\varepsilon>0$ be given and choose $\delta>0(\delta<\varepsilon)$ satisfying $2 \delta K e^{\int_{0}^{t_{0}} h(u) d u}+\alpha \varepsilon<$ $\varepsilon$. If $x(t)=x\left(t, t_{0}, \phi\right)$ is a solution of (2.1) with $\|\phi\|<\delta$, then $x(t)=(P x)(t)$ as defined in (2.11). We claim that $|x(t)|<\varepsilon$ for all $t \geq t_{0}$. Notice that $|x(s)|<\varepsilon$ on $\left[m\left(t_{0}\right), t_{0}\right]$. If there exists $t^{*}>t_{0}$ such that $\left|x\left(t^{*}\right)\right|=\varepsilon$ and $|x(s)|<\varepsilon$ for $m\left(t_{0}\right) \leq s<t^{*}$, then it follows from (2.11) that

$$
\begin{aligned}
\left|x\left(t^{*}\right)\right| \leq & \|\phi\|\left(1+\left|\frac{c\left(t_{0}\right)}{1-\tau^{\prime}\left(t_{0}\right)}\right|+\int_{t_{0}-\tau\left(t_{0}\right)}^{t_{0}}|h(s)-a(s)| d s\right) e^{-\int_{t_{0}}^{t^{*}} h(u) d u} \\
& +\varepsilon\left|\frac{c\left(t^{*}\right)}{1-\tau^{\prime}\left(t^{*}\right)}\right|+\varepsilon \int_{t^{*}-\tau\left(t^{*}\right)}^{t^{*}}|h(s)-a(s)| d s \\
& +\varepsilon \int_{t_{0}}^{t^{*}} e^{-\int_{s}^{t^{*}} h(u) d u} \mid-b(s)+[h(s-\tau(s))-a(s-\tau(s))]\left(1-\tau^{\prime}(s)\right) \\
& -\frac{\left[c(s) h(s)+c^{\prime}(s)\right]\left(1-\tau^{\prime}(s)\right)+c(s) \tau^{\prime \prime}(s)}{\left(1-\tau^{\prime}(s)\right)^{2}} \mid d s \\
+ & \varepsilon \int_{t_{0}}^{t^{*}} e^{-\int_{s}^{t^{*}} h(u) d u}|h(s)| \int_{s-\tau(s)}^{s}|h(v)-a(v)| d v d s \\
\leq & 2 \delta K e^{\int_{0}^{t_{0}} h(u) d u}+\alpha \varepsilon<\varepsilon
\end{aligned}
$$


which contradicts the definition of $t^{*}$. Thus $|x(t)|<\varepsilon$ for all $t \geq t_{0}$, and the zero solution of (2.1) is stable. This shows that the zero solution of (2.1) is asymptotically stable if (iii) holds.

Conversely, suppose (iii) fails. Then by (i) there exists a sequence $\left\{t_{n}\right\}, t_{n} \rightarrow \infty$ as $n \rightarrow \infty$ such that $\lim _{n \rightarrow \infty} \int_{0}^{t_{n}} h(u) d u=l$ for some $l \in R$. We may also choose a positive constant $J$ satisfying

$$
-J \leq \int_{0}^{t_{n}} h(s) d s \leq J
$$

for all $n \geq 1$. To simplify our expressions, we define

$$
\begin{aligned}
\omega(s) & =\mid-b(s)+[h(s-\tau(s))-a(s-\tau(s))]\left(1-\tau^{\prime}(s)\right) \\
& -\frac{\left[c(s) h(s)+c^{\prime}(s)\right]\left(1-\tau^{\prime}(s)\right)+c(s) \tau^{\prime \prime}(s)}{\left(1-\tau^{\prime}(s)\right)^{2}}|+| h(s)\left|\int_{s-\tau(s)}^{s}\right| h(v)-a(v) \mid d v
\end{aligned}
$$

for all $s \geq 0$. By (ii), we have

$$
\int_{0}^{t_{n}} e^{-\int_{s}^{t_{n}} h(u) d u} \omega(s) d s \leq \alpha
$$

This yields

$$
\int_{0}^{t_{n}} e^{\int_{0}^{s} h(u) d u} \omega(s) d s \leq \alpha e^{\int_{0}^{t_{n}} h(u) d u} \leq e^{J}
$$

The sequence $\left\{\int_{0}^{t_{n}} e^{\int_{0}^{s} h(u) d u} \omega(s) d s\right\}$ is bounded, so there exists a convergent subsequence. For brevity in notation, we may assume that

$$
\lim _{n \rightarrow \infty} \int_{0}^{t_{n}} e^{\int_{0}^{s} h(u) d u} \omega(s) d s=\gamma
$$

for some $\gamma \in R^{+}$and choose a positive integer $\bar{k}$ so large that

$$
\int_{t_{\bar{k}}}^{t_{n}} e^{\int_{0}^{s} h(u) d u} \omega(s) d s<\delta_{0} / 4 K
$$

for all $n \geq \bar{k}$, where $\delta_{0}>0$ satisfies $2 \delta_{0} K e^{J}+\alpha<1$.

By (i), $K$ in (2.9) is well defined. We now consider the solution $x(t)=x\left(t, t_{\bar{k}}, \phi\right)$ of (2.1) with $\phi\left(t_{\bar{k}}\right)=\delta_{0}$ and $|\phi(s)| \leq \delta_{0}$ for $s \leq t_{\bar{k}}$. An argument similar to that in (2.12) shows $|x(t)| \leq 1$ for $t \geq t_{\bar{k}}$. We may choose $\phi$ so that

$$
\phi\left(t_{\bar{k}}\right)-\frac{c\left(t_{\bar{k}}\right)}{1-\tau^{\prime}\left(t_{\bar{k}}\right)} \phi\left(t_{\bar{k}}-\tau\left(t_{\bar{k}}\right)\right)-\int_{t_{\bar{k}}-\tau\left(t_{\bar{k}}\right)}^{t_{\bar{k}}}[h(s)-a(s)] \phi(s) d s \geq \frac{1}{2} \delta_{0} .
$$


It follows from (2.11) with $x(t)=(P x)(t)$ that for $n \geq t_{\bar{k}}$,

$$
\begin{aligned}
& \left|x\left(t_{n}\right)-\frac{c\left(t_{n}\right)}{1-\tau^{\prime}\left(t_{n}\right)} x\left(t_{n}-\tau\left(t_{n}\right)\right)-\int_{t_{n}-\tau\left(t_{n}\right)}^{t_{n}}[h(s)-a(s)] x(s) d s\right| \\
& \quad \geq \frac{1}{2} \delta_{0} e^{-\int_{t_{\bar{k}}}^{t_{n}} h(u) d u}-\int_{t_{\bar{k}}}^{t_{n}} e^{-\int_{s}^{t_{n}} h(u) d u} \omega(s) d s \\
& \quad=\frac{1}{2} \delta_{0} e^{-\int_{t_{\bar{k}}}^{t_{n}} h(u) d u}-e^{-\int_{0}^{t_{n}} h(u) d u} \int_{t_{\bar{k}}}^{t_{n}} e^{\int_{0}^{s} h(u) d u} \omega(s) d s \\
& \quad=e^{-\int_{t_{\bar{k}}}^{t_{n}} h(u) d u}\left(\frac{1}{2} \delta_{0}-e^{-\int_{0}^{t_{\bar{k}}} h(u) d u} \int_{t_{\bar{k}}}^{t_{n}} e^{\int_{0}^{s} h(u) d u} \omega(s) d s\right) \\
& \quad \geq e^{-\int_{t_{\bar{k}}}^{t_{n}} h(u) d u}\left(\frac{1}{2} \delta_{0}-K \int_{t_{\bar{k}}}^{t_{n}} e^{\int_{0}^{s} h(u) d u} \omega(s) d s\right) \\
& \geq \frac{1}{4} \delta_{0} e^{-\int_{t_{\bar{k}}}^{t_{n}} h(u) d u} \geq \frac{1}{4} \delta_{0} e^{-2 J}>0 .
\end{aligned}
$$

On the other hand, if the zero solution of (2.1) is asymptotically stable, then $x(t)=$ $x\left(t, t_{\bar{k}}, \phi\right) \rightarrow 0$ as $t \rightarrow \infty$. Since $t_{n}-\tau\left(t_{n}\right) \rightarrow \infty$ as $n \rightarrow \infty$ and (ii) holds, we have

$$
x\left(t_{n}\right)-\frac{c\left(t_{n}\right)}{1-\tau^{\prime}\left(t_{n}\right)} x\left(t_{n}-\tau\left(t_{n}\right)\right)-\int_{t_{n}-\tau\left(t_{n}\right)}^{t_{n}}[h(s)-a(s)] x(s) d s \rightarrow 0 \quad \text { as } \quad n \rightarrow \infty,
$$

which contradicts (2.13). Hence condition (iii) is necessary for the asymptotic stability of the zero solution of (2.1). The proof is complete.

Remark 2.2. It follows from the first part of the proof of Theorem 2.1 that the zero solution of (2.1) is stable under (i) and (ii). Moreover, Theorem 2.1 still holds if (ii) is satisfied for $t \geq t_{\sigma}$ for some $t_{\sigma} \geq R^{+}$.

Remark 2.3. When $a(t) \equiv c(t) \equiv 0$, Theorem 2.1 with $h(s) \equiv b(g(s))$ reduces to Theorem B. On the other hand, we choose $h(s) \equiv a(s)$, then Theorem 2.1 reduces to Theorem C.

Remark 2.4. The method in this paper can be extended to the following general neutral differential equations with several variable delays:

$$
x^{\prime}(t)=-a(t) x(t)-\sum_{i=1}^{N} b_{i}(t) x\left(t-\tau_{i}(t)\right)+\sum_{j=1}^{M} c_{j}(t) x^{\prime}\left(t-\delta_{j}(t)\right) .
$$

\section{EXAMPLES}

Example 3.1. Consider the delay differential equation

$$
x^{\prime}(t)=-b(t) x(t-\tau(t)),
$$


where $\tau(t)=0.281 t, b(t)=\frac{1}{0.719 t+1}$. Following the notation in Theorem B, we have $b(g(t))=\frac{1}{t+1}$. Thus, as $t \rightarrow \infty$,

$$
\begin{gathered}
\int_{t-\tau(t)}^{t}|b(g(s))| d s=\int_{0.719 t}^{t} \frac{1}{s+1} d s=\ln \frac{t+1}{0.719 t+1} \longrightarrow-\ln (0.719), \\
\int_{0}^{t} e^{-\int_{s}^{t} b(g(u)) d u}|b(g(s))| \int_{s-\tau(s)}^{s}|b(g(v))| d v d s \\
=\frac{1}{t+1} \int_{0}^{t}[\ln (s+1)-\ln (0.719 s+1)] d s \\
=\ln (t+1)-\frac{t+1 / 0.719}{t+1} \ln (0.719 t+1) \longrightarrow-\ln (0.719) \\
\int_{0}^{t} e^{-\int_{s}^{t} b(g(u)) d u}|b(s)|\left|\tau^{\prime}(s)\right| d s=\frac{0.281}{t+1} \int_{0}^{t} \frac{s+1}{0.719 s+1} d s \\
=\frac{0.281}{0.719} \frac{t}{t+1}-\left(\frac{0.281}{0.719}\right)^{2} \frac{\ln (0.719 t+1)}{t+1} \longrightarrow \frac{0.281}{0.719}
\end{gathered}
$$

Thus, we have

$$
\begin{gathered}
\limsup _{t \geq 0}\left\{\int_{t-\tau(t)}^{t}|b(g(s))| d s+\int_{0}^{t} e^{-\int_{s}^{t} b(g(u)) d u}|b(g(s))| \int_{s-\tau(s)}^{s}|b(g(v))| d v d s\right. \\
\left.+\int_{0}^{t} e^{-\int_{s}^{t} b(g(u)) d u}|b(s)|\left|\tau^{\prime}(s)\right| d s\right\}=-2[\ln (0.719)]+\frac{0.281}{0.719}=1.0508 .
\end{gathered}
$$

In addition, the left-hand side of the following inequality is increasing in $t>0$, then there exists some $t_{0}>0$ such that for $t \geq t_{0}$,

$$
\begin{aligned}
& \int_{t-\tau(t)}^{t}|b(g(s))| d s+\int_{0}^{t} e^{-\int_{s}^{t} b(g(u)) d u}|b(g(s))| \int_{s-\tau(s)}^{s}|b(g(v))| d v d s \\
& +\int_{0}^{t} e^{-\int_{s}^{t} b(g(u)) d u}|b(s)|\left|\tau^{\prime}(s)\right| d s>1.05 .
\end{aligned}
$$

This implies that condition (2.5) does not hold. Thus, Theorem B cannot be applied to equation (3.1).

However, choosing $h(t)=\frac{1.2}{t+1}$, we have

$$
\begin{aligned}
\int_{t-\tau(t)}^{t} & |h(s)| d s=\int_{0.719 t}^{t} \frac{1.2}{s+1} d s=1.2 \ln \frac{t+1}{0.719 t+1}<0.396, \\
& \int_{0}^{t} e^{-\int_{s}^{t} h(u) d u}\left|-b(s)+h(s-\tau(s))\left(1-\tau^{\prime}(s)\right)\right| d s \\
& =\int_{0}^{t} e^{-\int_{s}^{t} \frac{1.2}{u+1} d u} \frac{1-1.2 \times 0.719}{0.719 s+1} d s \\
& <\frac{1-1.2 \times 0.719}{1.2 \times 0.719} \int_{0}^{t} e^{-\int_{s}^{t} \frac{1.2}{u+1} d u} \frac{1.2}{s+1} d s<0.1592
\end{aligned}
$$

and

$$
\int_{0}^{t} e^{-\int_{s}^{t} h(u) d u}|h(s)| \int_{s-\tau(s)}^{s}|h(v)| d v d s<0.396 .
$$

Let $\alpha:=0.396+0.396+0.1592=0.9512<1$, then the zero solution of $(3.1)$ is asymptotically stable by Theorem 2.1 . 
Example 3.2. Consider the neutral differential equation

$$
x^{\prime}(t)=-a(t) x(t)+c(t) x^{\prime}(t-\tau(t)),
$$

where $a(t)=\frac{1}{t+1}, \tau(t)=0.05 t, c(t)=0.48$. Obviously,

$$
\begin{aligned}
& \left|\frac{c(t)}{1-\tau^{\prime}(t)}\right|+\int_{0}^{t} e^{-\int_{s}^{t} a(u) d u}\left|\frac{\left[c(s) a(s)+c^{\prime}(s)\right]\left(1-\tau^{\prime}(s)\right)+c(s) \tau^{\prime \prime}(s)}{\left(1-\tau^{\prime}(s)\right)^{2}}\right| d s \\
& =\frac{0.48(2 t+1)}{0.95(t+1)} .
\end{aligned}
$$

Since the right-hand side of (3.3) is increasing in $t>0$ and

$$
\limsup _{t \geq 0}\left\{\frac{0.48(2 t+1)}{0.95(t+1)}\right\}=1.0105
$$

then there exists some $t_{0}>0$ such that $t \geq t_{0}$,

$$
\left|\frac{c(t)}{1-\tau^{\prime}(t)}\right|+\int_{0}^{t} e^{-\int_{s}^{t} a(u) d u}\left|\frac{\left[c(s) a(s)+c^{\prime}(s)\right]\left(1-\tau^{\prime}(s)\right)+c(s) \tau^{\prime \prime}(s)}{\left(1-\tau^{\prime}(s)\right)^{2}}\right| d s>1.01 .
$$

This implies that condition (2.8) does not hold. Thus, Theorem $\mathrm{C}$ cannot be applied to equation (3.2).

However, choosing $h(t)=\frac{2.2}{t+1}$, we have

$$
\begin{gathered}
\left|\frac{c(t)}{1-\tau^{\prime}(t)}\right|<0.506 \\
\int_{t-\tau(t)}^{t}|h(s)-a(s)| d s=\int_{0.95 t}^{t} \frac{1.2}{s+1} d s=1.2\left[\ln \left(\frac{t+1}{0.95 t+1}\right)\right]<0.062, \\
\int_{0}^{t} e^{-\int_{s}^{t} h(u) d u}|h(s)| \int_{s-\tau(s)}^{s}|h(v)-a(v)| d v d s<0.062
\end{gathered}
$$

and

$$
\begin{aligned}
& \int_{0}^{t} e^{-\int_{s}^{t} h(u) d u \mid[h(s-\tau(s))-a(s-\tau(s))]\left(1-\tau^{\prime}(s)\right)} \\
& \quad-\frac{\left[c(s) h(s)+c^{\prime}(s)\right]\left(1-\tau^{\prime}(s)\right)+c(s) \tau^{\prime \prime}(s)}{\left(1-\tau^{\prime}(s)\right)^{2}} \mid d s \\
& =\int_{0}^{t} e^{-\int_{s}^{t} \frac{2.2}{u+1} d u}\left(\frac{1.2 \times 0.95}{0.95 s+1}-\frac{2.2 \times 0.48}{0.95(s+1)}\right) d s<\frac{1.2}{2.2}-\frac{0.48}{0.95}=0.041 .
\end{aligned}
$$

Let $\alpha:=0.506+0.062+0.062+0.041=0.671<1$, then the zero solution of $(3.2)$ is asymptotically stable by Theorem 2.1 .

\section{ACKNowledgments}

The authors thank very sincerely the anonymous referees for their valuable comments and helpful suggestions.

\section{REFERENCES}

1. T. A. Burton, Stability and Periodic Solutions of Ordinary and Functional Differential Equations, Academic Press, New York, 1985. MR837654 (87f:34001)

2. T. A. Burton, Liapunov functionals, fixed points, and stability by Krasnoselskii's theorem, Nonlinear Studies, 9 (2001), 181-190. MR1898587 (2003e:34133) 
3. T. A. Burton, Stability by fixed point theory or Liapunov's theory: a comparison, Fixed Point Theory, 4 (2003), 15-32. MR2031819 (2004j:34110)

4. T. A. Burton, Fixed points and stability of a nonconvolution equation, Proc. Amer. Math. Soc., 132 (2004), 3679-3687. MR2084091 (2005g:34186)

5. T. A. Burton and T. Furumochi, A note on stability by Schauder's theorem, Funkcialaj Ekvacioj, 44 (2001), 73-82. MR1847837 (2002d:34075)

6. T. A. Burton and T. Furumochi, Fixed points and problems in stability theory, Dynamical Systems and Appl., 10 (2001), 89-116. MR1844329 (2002c:34076)

7. T. A. Burton and T. Furumochi, Krasnoselskii's fixed point theorem and stability, Nonlinear Analysis, 49 (2002), 445-454. MR1886230 (2003e:34087)

8. Y. N. Raffoul, Stability in neutral nonlinear differential equations with functional delays using fixed-point theory, Mathematical and Computer Modelling, 40 (2004), 691-700. MR2106161

9. B. Zhang, Fixed points and stability in differential equations with variable delays, Nonlinear Analysis, 63 (2005), e233-e242.

Faculty of Applied Mathematics, Guangdong University of Technology, Guangzhou, Guangdong 510090, People's Republic of China

E-mail address: jinchuhua@tom.com

Corresponding author. School of Mathematics and Information Science, Guangzhou University, Guangzhou, Guangdong 510006, People's Republic of China

E-mail address: mathluo@yahoo.com 